\title{
Energy efficient solutions for the networks and routers
}

\author{
Xing Zheng ${ }^{1}$, Lingling Sun ${ }^{1}$, Jie Jin ${ }^{2}$, Xiaojun Wang ${ }^{2}$ \\ 1.Key Laboratory of RF Circuits and Systems of Ministry of Education, Hangzhou Dianzi University, \\ Hangzhou, 310018, China \\ 2.School of Electronic Engineering, Dublin City University, Dublin 9, Ireland
}

Corresponding author: zhengxing@hdu.edu.cn

Keywords: energy efficiency, networks and routers, frequency scaling, publish/subscribe.

\begin{abstract}
Under the pressure of the global CO2 emission, energy efficiency is one of the most important aspects that need considerations in designing the future Internet. For reducing the power consumption of the Internet, people should focus their research attentions on the power-aware networks design and most power consuming networking devices such as routers. This paper overviews a number of promising solutions in recent years for reducing the energy consumption of the networks and routers. Moreover, we present our research achievement about an energy efficient networking forwarding node on the NetFPGA platform by combining the salient advantages of the Bloom filter based publish/subscribe forwarding node and frequency scaling. Our findings show that the frequency scalable Bloom filter (zFilter) forwarding node can not only improve the forwarding performance, but also considerably reducing the power consumption of the router.
\end{abstract}

\section{Introduction}

In the past few decades, the Internet has developed extremely fast [1]. The large growth in the number of end hosts and the network speeds makes the Internet experience exponential bandwidth scaling. However, the increase in the network bandwidth is at the cost of increasing the network power consumption. The larger the network becomes, the more the power it consumes. Today, the telecommunication industry is responsible for approximately five percent of the overall power consumption in the developed nationals [2]. The Internet consumes about one percent of the total electrical power in the developed countries. The open literatures estimated that $[3,4]$, the total network energy requirement for European telecom operators (Telcos) will reach 35.8TWh in 2020 if there is no Green technology used.

For realizing the energy efficiency of the Internet, research attentions have been given to the approaches for reducing the power consumption of the networks and the most power-consuming networking components such as routers. The proposed green technologies includes [1-3,5] for example: (1) Re-engineering approaches; (2) sleeping and smart standby; (3) the use of optical components in the router; (4) dynamically adapting the router capacity to the current throughput; (5) energy efficient network design; (6) frequency scaling.

This paper overviews the above-mentioned power aware approaches. For further investigating the energy efficient schemes, we move our attention to a publish/subscribe forwarding node called zFilter. The zFilter is a Bloom filter based forwarding node, proposed and implemented in publish/subscribe networks [6]. It is more faster forwarding than the current IP based routing scheme due to its very simple forwarding decisions. Therefore, we present in this paper about our research achievements: a frequency scalable zFilter which combines the advantages of the zFilter forwarding node and the frequency scaling technique. It is shown that the frequency scaling zFilter can improve the forwarding performance significantly and more importantly reduce the power consumption of the router considerably.

The rest of the paper is organized as follows. Section 2 overviews the current power aware approaches. Section 3 describes the proposed frequency scaling zFilter and our experimental testing environment. Section 4 presents the experimental results. The paper is finally drawn in Section 5. 


\section{Current approaches to reduce the power consumption of the routers and networks}

\subsection{Re-engineering approaches:}

Re-engineering approaches aims at reducing the complexity of device internal architectures, optimizing the internal organization devices and using more energy-efficient hardware techniques. By using this approach, the functionalities of the router can be significantly reduced and the router can be manufactured with a lower amount of hardware logical gates. One good example of the reengineering approaches is the radical new router concept proposed by [5,7]. In this new scheme, the router forwards traffic at the flow level, which allows to a more scalable and simple network device architecture compared with the current routers forwarding traffic at the packet level.

\subsection{Sleeping and smart standby}

Sleeping and smart standby are the approaches in which the whole devices or part of them are almost turned off and entering into the low energy mode [5]. In this case, all or most of the device functionalities are frozen and only the basic functionalities are performed. Then, the devices or part of them are waken up only if necessary. IEEE 802.3az energy efficient Ethernet (EEE) standard [8] is a good example of the sleeping and smart standby approaches. In EEE, when there is no data or traffic exchanged in a link, the physical layer can be switched into a lower energy mode and the power consumption of the networks can be greatly reduced. Once the data or traffic arrives, the transmission link is activated. The transition time is in the order of microseconds and therefore the added time delay is negligible to many applications. Thus, the ports/ linecards/ whole router can be turned off when the router is entering into the low energy mode.

However, sleeping and smart standby approaches are also facing some problems. One major problem is that when a device or part of it goes into the sleeping mode, its applications stop working and thus lose their network connectivity. As a direct result, the sleeping device loses its network and can not answer to application or service-specific messages. Moreover, the device has to re-initialize its applications when it wakes up and the device also needs to send a non-negligible amount of signalling traffic.

\subsection{The use of optical components in the router}

Optical components can provide higher bandwidth and consume less power than the electronic device [1]. For example, the optical switch is able to support the bandwidth of terabits per second with very low power consumption. Therefore in the telecommunication industry, it is considered that for long term solution, the optical switch can replace the electronic router. Many research institutions and universities also focus on this energy efficient scheme for the router. For example, the NetFPGA 10G which is a hardware accelerate research platform also involves the optical components into the $10 \mathrm{G}$ router and switch architectures.

\subsection{Dynamically adapting the router capacity to the current throughput}

The dynamic adaptation of network or router is the approach that modulates the capacities of network device resources such as packet processing engines and network interfaces and finally to meet actual traffic loads and service requirements. This can be performed by using a number of power-aware schemes including for example dynamic voltage (power) scaling and idle logic. These schemes allow the dynamic trade-off between the packet service performance and power consumption of the routers.

\subsection{Energy efficient network design}

For implementing high capacity and reliable networks, the network manufacturers traditionally put most of the functionalities in the core network routers. This accompanies with the high power consumption. The long term goal of the network design is to replace the power hungry routers with the power aware routers while still maintain the network capacity, reliability, robustness and performance. 
For achieving a reliable routing, there are redundant paths in the existing networks. Another approach to reduce the power consumption is thus to redesign the network topology and reorganize the network structure which can minimize the network power consumption. Moreover, the networks can also be designed using the following way that the most power consuming operations are limited to several routers [1].

\subsection{Frequency scaling}

The frequency scaling approach is adopted by the academics and industries in order to reduce the power consumption of the routers. The idea behind this approach is that the logic clock of the main chip or core FPGA can be switched into lower frequency and thus the total power consumption of the main chip or core FPGA can be reduced significantly. The drawback of this approach is that when the core operational frequency of the router is scaled down, the forwarding capacity is also reduced. Therefore, the adoption of this approach follows the principle that the router can be down scaled to lower frequency if the traffic is small and up scaled to higher frequency if the traffic is big.

\section{Energy efficient publish/subscribe forwarding node}

\subsection{The zFilter forwarding mechanism and implemented frequency scaling zFilter}

For enabling an energy efficient routing and forwarding device, we use the Bloom filter-based publish/subscribe forwarding node (proposed and implemented firstly by [6]). This forwarding node is called "zFilter". The motive of proposing this forwarding is originally to realize an informationcentric networks (ICN) node.

In the recent years, researches have moved their attention to the ICN, where the users are only interested in accessing information, instead of its physical location. Compared to host-centric networks which are commonly based on the end-to-end IP scheme, ICN has a number of salient advantages including, for example, efficient access and distribution of information, mobility support, and better network security. As one type of ICN, the publish/subscribe networks has attracted lots of research interest.

For realizing the publish/subscribe networks, zFilter is a feasible solution. The zFilter is a publish/subscribe forwarding node which is realized by using a Bloom filter-based matching approach. This approach uses link identification instead of node identification. Each zFilter forwarding node contains a Link ID per port. The Link ID is used to identify a link in each direction. By encoding all the Link IDs relating with the delivery, the forwarding information can be constructed into a Bloom filter. The Bloom filter is then inserted to the transmitted packet header to form the zFilter.

Once the zFilter forwarding node receives a packet, it performs a logical AND operation between the zFilter forwarding information in the packet header and the outgoing Link ID of each link. Then, the zFilter carries on a comparison operation between the final result of the AND operation and the outgoing Link ID of each link. When matching occurs after the comparison, the packet is forwarded out on that matching link. Detailed descriptions of the Bloom filter scheme and the zFilter can be found in [6]. The forwarding decision of the zFilter is much simpler than the IPv4 router such that it can offer better forwarding performance than the IPv4 router.

It is known that the frequency down scaling can reduce the power consumption, which however inevitably lowers the forwarding capacity of the router or other routing device. Fortunately, we found zFilter can overcome this drawback. Therefore, we implement the frequency scaling feature on the zFilter forwarding node by using the NetFPGA platform. Based on the open source project of zFilter, we introduce digital clock manager (DCM) and multiplexed global clock buffer (BUFGMUX) into the zFilter architecture. As shown in Fig.1, the DCM can divide the internal source clock, synthesizes and generates new frequencies by using frequency division functionality. After that, the generated new frequency is input into the BUFGMUX, drives the global clock resource and finally distributes the new frequency to most of the modules within the zFilter forwarding node. 


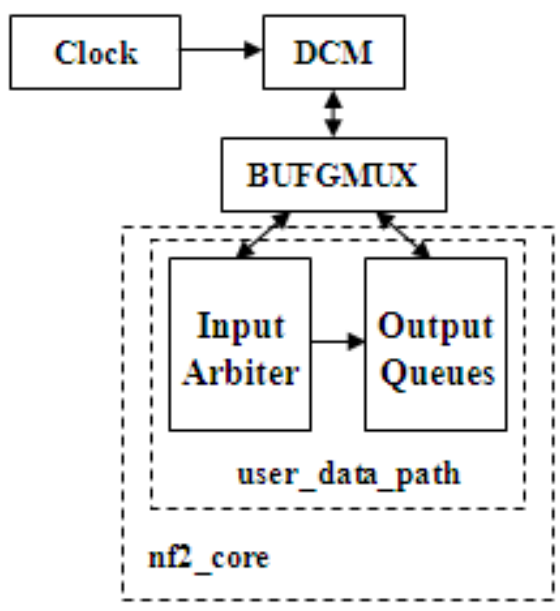

Fig.1: The architecture of the frequency scaling zFilter forwarding node.

\subsection{The experimental setup}

We build a real time testing environment for measuring the power consumption and the forwarding performance of the NetFPGA-enabled routers. The testing environment consists of a zFilter (or IPv4 router), a packet generator and an energy measurement tool kit. One NetFPGA board is configured as a zFilter or IPv4 router. The core operational frequency can be toggled between three different frequencies including $125 \mathrm{MHz}, 62.5 \mathrm{MHz}$ and $41.7 \mathrm{MHz}$. The packet generator connects with the zFilter (or IPv4 router), which performs as the traffic source and sink. The packet generator is implemented by using a second NetFPGA board. The packet generator can create a packet stream and adjustable data rate at each port.

The power consumption of the zFilter (or IPv4 router) is measured through the combination of a PCI bus extender [9], a National Instruments data acquisition (NI DAQ) device [10] and the LabVIEW software. The PCI bus extender is plugged into the motherboard slot of one host PC and the NetFPGA zFilter (or IPv4 router) is inserted into the PCI bus extender. The NI DAQ is connected to the PCI bus extender for measuring the current from the $5 \mathrm{~V}$ pin and the $3.3 \mathrm{~V}$ pin. The total power consumption can be obtained by accumulating the power from the $5 \mathrm{~V}$ and $3.3 \mathrm{~V}$ pins. For collecting the power data from the NI DAQ, LabVIEW running on another separate PC is used. The NI DAQ is connected to this PC through a USB link.

\section{The new findings and results}

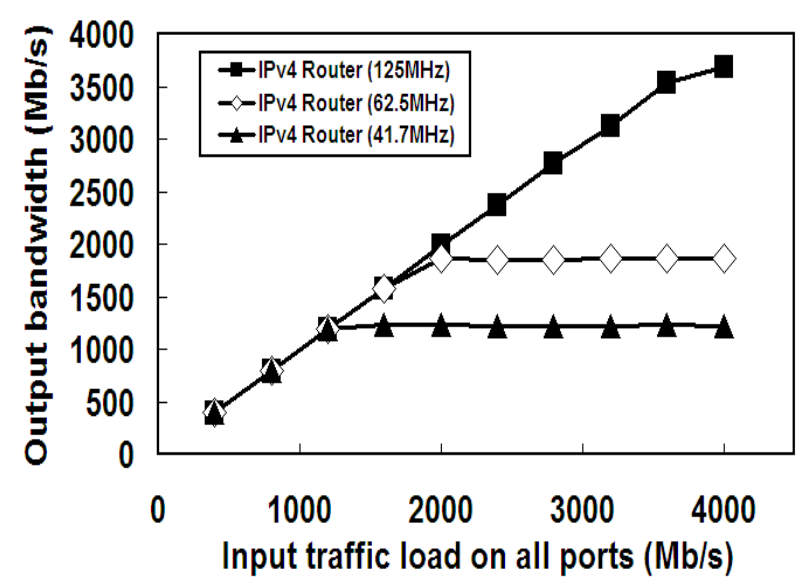

(a) IPv4 router

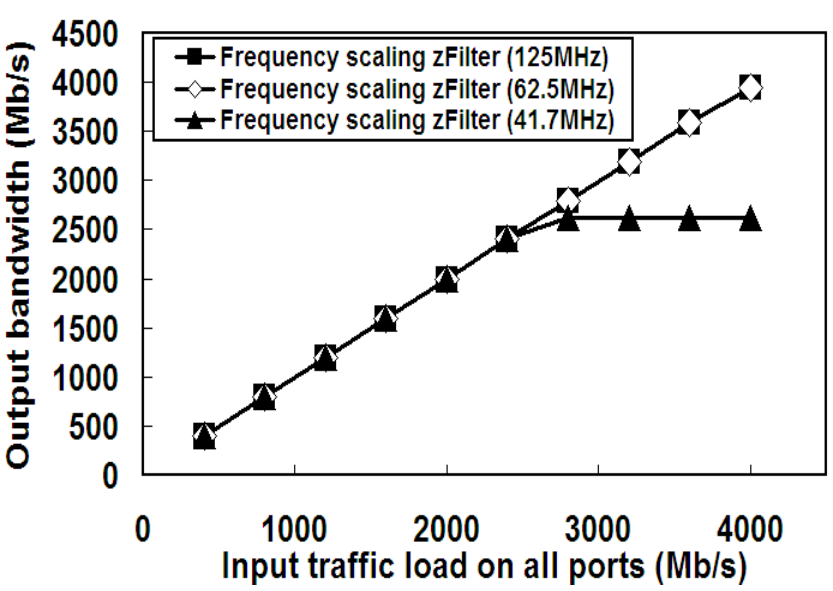

(b) zFilter

Fig.2: The forwarding performance for the zFilter and IPv4 router under different operating frequencies. 


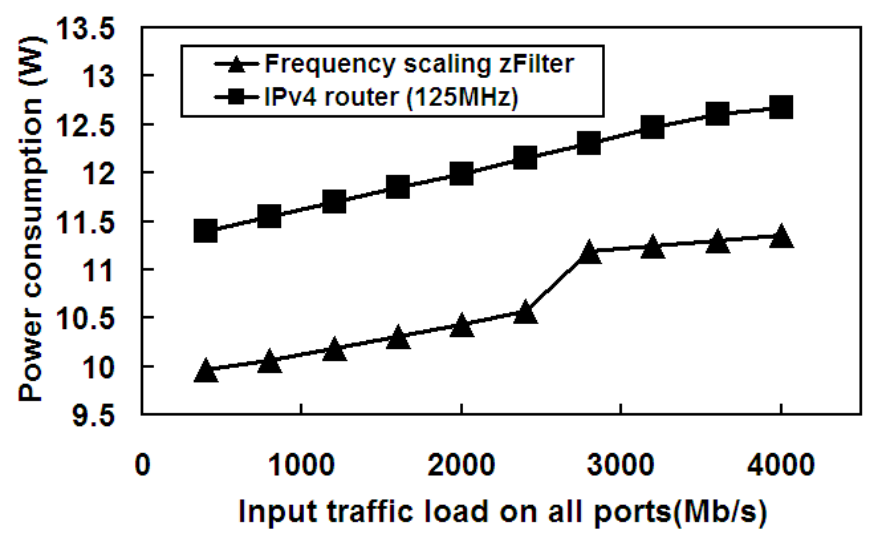

Fig.3: Power consumption reduction of the zFilter.

The frequency down scaling causes the worse of the forwarding performance for the IPv4 router. It can be found in Fig.2(a) that (1) 50\% down scaling the frequency from $125 \mathrm{MHz}$ more than halves the forwarding capacity of the IPv4 router; (2) further reducing the operating frequency to $41.7 \mathrm{MHz}$ results in less than $1300 \mathrm{Mb} / \mathrm{s}$ for the maximum achievable forwarding capacity.

Fortunately, such performance can be significantly improved by using the frequency scaling zFilter. It can be seen in Fig.2 (b) that by reducing the operating frequency to $62.5 \mathrm{MHz}$, there is no forwarding performance degradation for the zFilter. Even further reducing the operating frequency to $41.7 \mathrm{MHz}$, the frequency scaling zFilter can also achieve more than $2600 \mathrm{Mb} / \mathrm{s}$ for the maximum forwarding capacity.

Therefore, the frequency scaling can operate at lower frequency level while still maintains a better forwarding performance than the IPv4 router. As shown in Fig.3, by adjusting the operating frequency to $62.5 \mathrm{MHz}$ and $41.7 \mathrm{MHz}$, the frequency scaling zFilter can reduce the power consumption by at most $15.0 \%$. This indicates that our proposed frequency scaling zFilter forwarding node has the great potential for enabling an energy efficient forwarding and routing device in the future Internet.

\section{Conclusions}

This paper summarizes the current promising approaches for reducing the energy consumption of the networks and routers. We also present our research achievements about an energy efficient forwarding node on the NetFPGA platform. This forwarding node combines the advantages of the Bloom filter based publish/subscribe forwarding node and frequency scaling technique. Our results show that our frequency scaling Bloom filter (zFilter) forwarding node can significantly improve the forwarding performance, and more importantly considerably reducing the power consumption of the router.

\section{Acknowledgements}

This work was supported by Zhejiang Provincial Natural Science Foundation of China (ZJNSF) (Grant Number: LQ13F010009), Zhejiang Provincial Qianjiang Talent Plan (QJD1402001), National Natural Science Foundation of China (Grant Number: 61331006), Science Foundation Ireland under the International Strategic Cooperation Award (Grant Number: SFI/12/ISCA/2496) and the Program for Zhejiang Provincial Leading Team of Science and Technology Innovation (2010R50010).

\section{References}

[1] Chabarek J., Sommers J., Barford P., Estan C., Tsiang D. \& Wright S., "Power awareness in network design and routing,” Proc. IEEE INFOCOM, USA, pp. 1130-1138, 2008.

[2] Hinton K., Baliga J., Feng M., Ayre R. \& Tucker R. S., "Power consumption and energy efficiency in the Internet,” IEEE Network, 25 (2), pp. 6-12, 2011. 
[3] Vishwanath A., Zhao Z., Sivaraman V. \& Russell C., "An empirical model of power consumption in the NetFPGA gigabit router," Proc. IEEE Int.l Symp. Adv. Network. Telecommun. Syst. (ANTS), India, pp. 16-18, 2010.

[4] Bolla R., Davoli F., Bruschi R., Christensen K., Cucchietti F. \& Singh S., “The potential impact of green technologies in next-generation wireline networks - is there room for energy saving optimization?” IEEE Commun Mag, 49(8), pp. 80-86, 2011.

[5] Bolla R., Bruschi R., Davoli F. \& Cucchietti F., "Energy efficiency in the future Internet: a survey of existing approaches and trends in energy-aware fixed network infrastructures,” IEEE Communications Surveys and Tutorials, 13(2), pp.223-244, 2011.

[6] Keinanen J., Jokela P. \& Slavov K., "Implementing zFilter based forwarding node on a NetFPGA,” NetFPGA Developers Workshop, 2009.

[7]. Roberts L. G, “A Radical New Router,” IEEE Spectrum, 46(7), pp. 34-39, 2009.

[8] Reviriego P., Christensen K., Rabanillo J. \& Maestro J. A., “An initial evaluation of energy efficient Ethernet,” IEEE Commun Lett, 15(5), pp. 578-580, 2011.

[9] Ultraview PCI Smart Extenders: www.ultraviewcorp.com

[10] National Instruments NI USB-6251: www.ni.com 